Editorial

\title{
Emerging Technologies: IoT, Big Data, and CPS with Sensory Systems
}

\author{
Qing Tan $\mathbb{D}^{1},{ }^{1}$ Nashwa El-Bendary $\mathbb{D}^{2}{ }^{2}$ Magdy A. Bayoumi, ${ }^{3}$ Xiaokun Zhang, \\ Javier Sedano $\mathbb{D}^{4}{ }^{4}$ and José R. Villar ${ }^{5}{ }^{5}$ \\ ${ }^{1}$ School of Computing and Information Systems, Athabasca University, Athabasca, AB, Canada \\ ${ }^{2}$ Arab Academy for Science, Technology, and Maritime Transport, Smart Village, Giza, Egypt \\ ${ }^{3}$ University of Louisiana at Lafayette, Lafayette, LA, USA \\ ${ }^{4}$ Instituto Tecnológico de Castilla y León, Polg. Ind. Villalonquejar, López Bravo 70, 09001 Burgos, Spain \\ ${ }^{5}$ Computer Science Department, University of Oviedo, EIMEM, cl Independencia 13, 33004 Oviedo, Spain
}

Correspondence should be addressed to Qing Tan; qingt@athabascau.ca

Received 5 December 2017; Accepted 5 December 2017; Published 18 February 2018

Copyright (C) 2018 Qing Tan et al. This is an open access article distributed under the Creative Commons Attribution License, which permits unrestricted use, distribution, and reproduction in any medium, provided the original work is properly cited.

This collection of studies is focused on a three-legged stand in which now is focused on the research community: the internet of things (IoT), the cyber-physical systems (CPS), and the data-driven knowledge extraction based on big data. The availability of uniquely addressable heterogeneous electronic (UAHE) - including sensors, actuators, smart devices, RFID tags, embedded computers, and mobile devices-is continuously growing day by day. From a networking perspective, the IoT relies on interconnected UAHE for creating a mesh of devices, producing information, and building a worldwide network of real physical objects. In this context, the IoT presents a technology that enables loosely coupled decentralized systems of cooperating smart objects of autonomous physical-digital devices, augmented with sensing/ actuating, processing, and networking capabilities.

Over the past 17 years, since the term IoT was first coined by Kevin Ashton in 1999, radio frequency identification (RFID) system is shaping up to be an important building block and a prerequisite for the IoT. The RFID is a key technology that automatically utilizes devices to identify, track and monitor, or control objects through radio waves. However, the performance of RFID equipment can be easily affected by factors in the surrounding environment. Consequently, that can impose a negative impact on the RF signal and the source data. $\mathrm{H}$. Xu et al. proposed a sliding window cleaning algorithm called VSMURF, which is based on the traditional SMURF algorithm. In this research, the algorithm they proposed combines the dynamic change of RFID tags and the value analysis of confidence $(\delta)$ in order to solve the false negative problems affecting the quality of the collected data. Also, they used only one RFID reader and 25 tags for their experimental setting. The obtained experimental results showed that their proposed VSMURF algorithm outperformed the typical SMURF algorithm in most conditions, and when the tag's speed is low or high.

However, the frontiers between IoT and CPS are not always well defined. Some real world problems, like those related with eHealth and health monitoring, are in between: on the one hand, they gather information from the user environment with the aim of delivering the advice to either the user, the relatives, or the medical staff. P. M. Vergara et al. analyzed this problem in a real context of the epilepsy seizure detection. This research studies the problem of a system that, using wearable devices and Smartphones, copes with the detection of tonic-clonic seizures. The main factors in the design issues for such a platform are detailed. Finally, a description of a platform that is being developed is detailed, providing an experimentation on the robustness of the data communications. This experimentation would eventually 
help in the online task distribution among the different computational resources.

Another example of the difficulty in establishing the limits of IoT and CPS is the problem of mobile crowd sensing: that is, using the sensory systems carried by each individual in a population in order to extract some relevant conclusion on a specific topic, like environment monitoring and positioning. No need to argue, this big data challenge as well as the problem of choosing the features, and how to perform the data fusion, and so on are topics covered with big data techniques. Nevertheless, L.-Y. Jiang et al. studied the problem of the trust ability of the data as a problem of avoiding individuals to introduce false data-either intentionally or unintentionally. Furthermore, the authors propose an incentive system to tackle this issue. The proposed incentive system is based on the conditions of recruiters and candidates, becoming in a quality assessment optimization problem solved by a polynomial-time greedy approximation algorithm the authors proposed. The simulation-based experimentation is complete and full of interest.

One of the problems that the IoT research community needs to tackle is to ensure the security: not only the problems concerning the unauthorized and/or destructive actions on the devices and/or infrastructure but also those related with cyberattacks and any kind of malicious strikes. Surprisingly, this issue has not been studied in depth due to the variability of the IoT scenarios: different communication hardware and software, the computation restrictions in the nodes, and so on. This issue has been analyzed in T. Andrysiak et al., proposing several interesting concepts that need to be considered when dealing with security issues. Furthermore, the authors proposed a very interesting method for detecting anomalies in the network traffic that might arise from cyberattacks. To do so, the authors proposed to estimate the normal network behaviour by (i) filtering outliers; (ii) computing the exponential smoothing models, either with Brown's, Holt's, or Winter's models; (iii) analysing the fluctuations of the estimations using Bollinger's bands; and (iv) comparing the obtained estimations of normal behaviour with what is really happening in the network. A very complete experimentation is performed using smart metering networks, a real IoT problem indeed.

Besides, challenges in the deployment of IoT solutions are also focused on energy efficiency. In passive backscatter communication systems, sensor nodes need to harvest RF energy from transceivers or readers and use it to recharge their finite energy storage capacity. However, RF energy harvesting also faces unpredictable environmental challenges, which make the sensors' sensing, processing, and communication activities difficult. Therefore, an efficient energy management is necessary in order to guarantee the sensors' activities and QoS (quality of service) of backscatter communication. In this special issue, the article contributed by $\mathrm{S}$. Hu et al. present an RF energy harvesting and a colocated passive quadrature amplitude modulation (QAM) backscatter communication signal models. The proposed models aim at achieving good quality of service (QoS) level through minimizing interference resulted from multisensor and increasing spectral efficiency. Experimental results, based on numerical analysis and simulations, showed that the maximum throughput inversely relates to the consumed power and the number of sensors. Also, for a given consumed power of sensors, it was observed that the throughput decreases with duty cycle, and the number of sensors has little effect on the throughput.

On the other hand, the coupling between the IoT and the big data communities is strong as big data analytics has become an essential component for extracting value from data. Big data as one of the most important and recent research challenges with a paradigm relies on the collection of tremendous amount of data to support innovation in the upcoming decades. A dataset is considered as big data when it meets the "four Vs" requirements, namely, volume, variety, velocity, and value. The keystone of big data exploitation is to leverage the existing datasets to create new information and predict future happenings, enriching the decision value chain. Accordingly, as the IoT continuously collects data about the surrounding living environments, it is considered as a prototypical example of big data and a great application area of big data analytics.

Dealing with large amount of dynamic data is one of important characteristics of big data analytics. Entity resolution (ER) is to disambiguate manifestations of real-world entities in the dataset by linking and grouping and to reduce the complexity of data processing, which is a crucial step to achieve effective, efficient, and accurate data processing in big data analysis. H.-J. Zhu et al. proposed a type-based multiblock technique to improve data quality. In the article, they present a new ER solution using a hybrid approach. In particular, through their new ER workflow, it is capable to reduce the searching space for entity pairs. The performance of their proposed method has been validated through using a real-life dataset created from an IoT real project. These results have been obtained using up to five standard metrics. The experimental results conclude that their proposed approach can be a promising alternative for ER over large-scale data.

Besides, CPS are emerging from the integration of embedded computing devices, smart objects, people, and physical environments, which are typically tied by a communication infrastructure. So, the design of CPS and the implementation of their applications need to rely on IoTenabled architectures and protocols that, both locally and globally, enable collecting, managing, and processing large data sets and support complex processes to manage and control such systems. Thus, as a matter of fact, the large-scale nature of IoT-based CPS can be effectively and efficiently facilitated and supported via utilizing the cloud computing infrastructures and platforms for providing flexible computational power, resource virtualization, and high-capacity storage for data streams in addition to ensuring safety, security, and privacy.

CPS are the extension of traditional closed-loop systems with the computer-based control and the Internet-based communication, in which physical components, computer algorithms, and sensing elements are tightly integrated via the Internet communication link. Sensors are the essential 
elements in any closed-loop system. In this special issue, the article contributed by L. Sanchez et al. present a model-based virtual sensor to be used for Li-Ion batteries' condition monitoring in cyber-physical vehicle systems. A principlebased model encoded with the expert knowledge about battery behaviour has been turned into a soft sensor. Feeding with the information extracted from data collected from on-vehicle measurements, the soft sensor is able to approximate the state of health of a battery. The experimental results coming from the implementation of the model-based soft sensor for fault detection and diagnosis of the batteries have demonstrated the soft sensor's high efficiency.

As an example of CPS, W. Yu et al. proposed a new approach to generate flexible gait for bionic leg's control based on muscle synergies extracted from sEMG (surface electromyogram) signal. The approach was inspired by the fact that muscle synergies leading to dimensionality reduction may simplify motor control and learning. Usually, typical multichannel sEMG signal-based controls need mass data and vary greatly with time which cause worse latency and other performance-related issues and make it difficult to generate compliant gait. The paper addresses two questions to highlight the essential features in the proposed approach with an interesting experimental result. The first question addresses whether the same set of muscle synergies can explain the different phases of jumping movement with various velocities. The second question is about building a model for generating velocity-adapted jumping gait with muscle synergies, in which a wavelet neural network is proposed to predict the reference gait pattern, while fuzzy inference system is adopted to merge these reference gaits in order to create more generalized gaits with different jumping rhythms. The proposed method can be adopted as the decoder in sEMG-based controls for a bionic leg. Moreover, linear combinations of synergies may describe complex force and motion patterns in reduced dimensions, and the robust representations of synergies within the control scheme can generate flexible gaits for other complex motions.

\author{
Qing Tan \\ Nashwa El-Bendary \\ Magdy A. Bayoumi \\ Xiaokun Zhang \\ Javier Sedano \\ José R. Villar
}




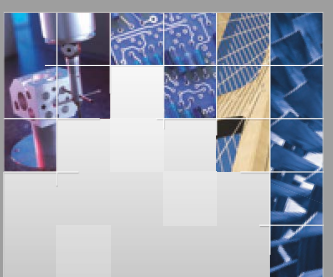

\section{Enfincering}
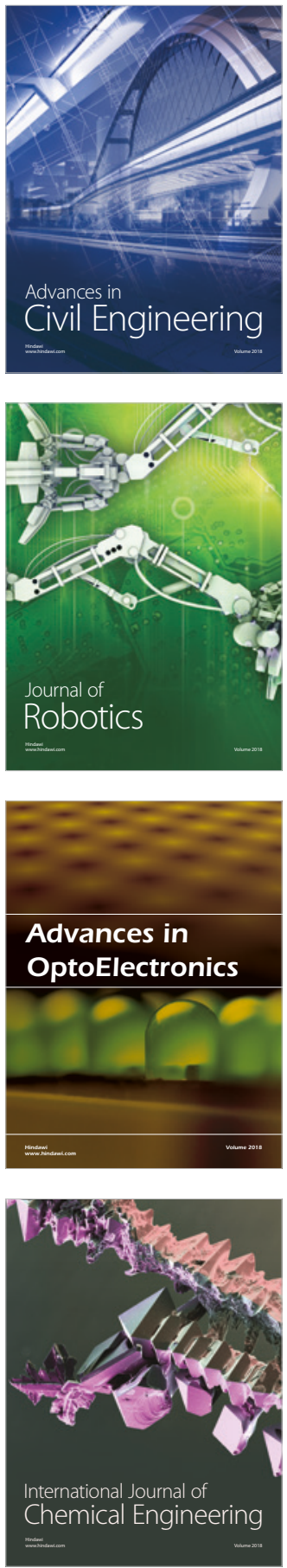

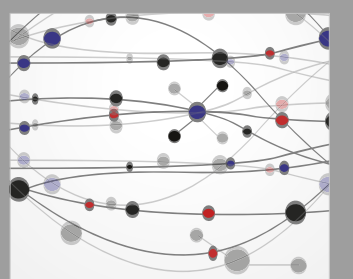

\section{Rotating \\ Machinery}

The Scientific World Journal

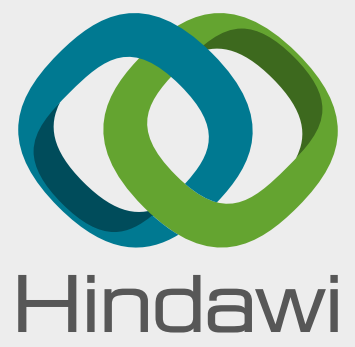

Submit your manuscripts at

www.hindawi.com
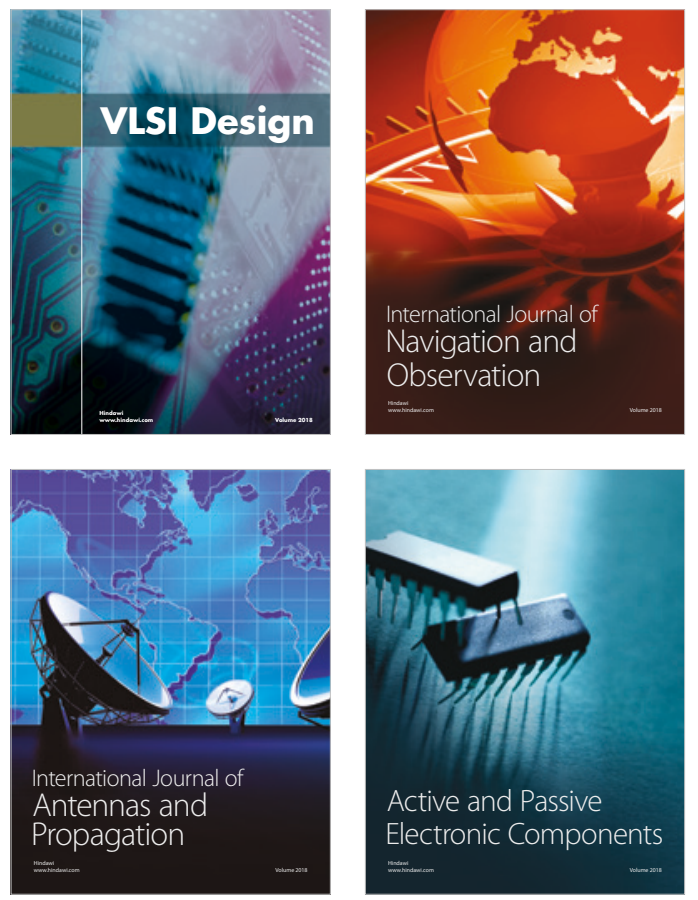
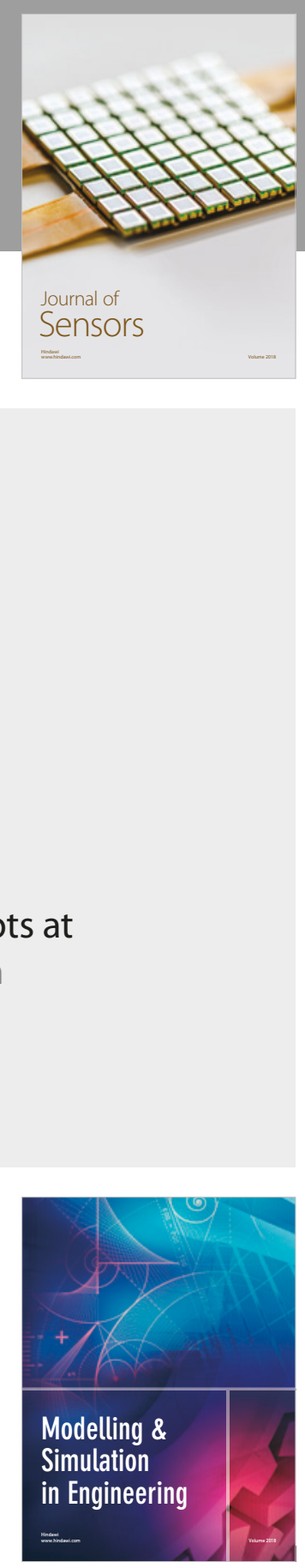

\section{Advances \\ Multimedia}
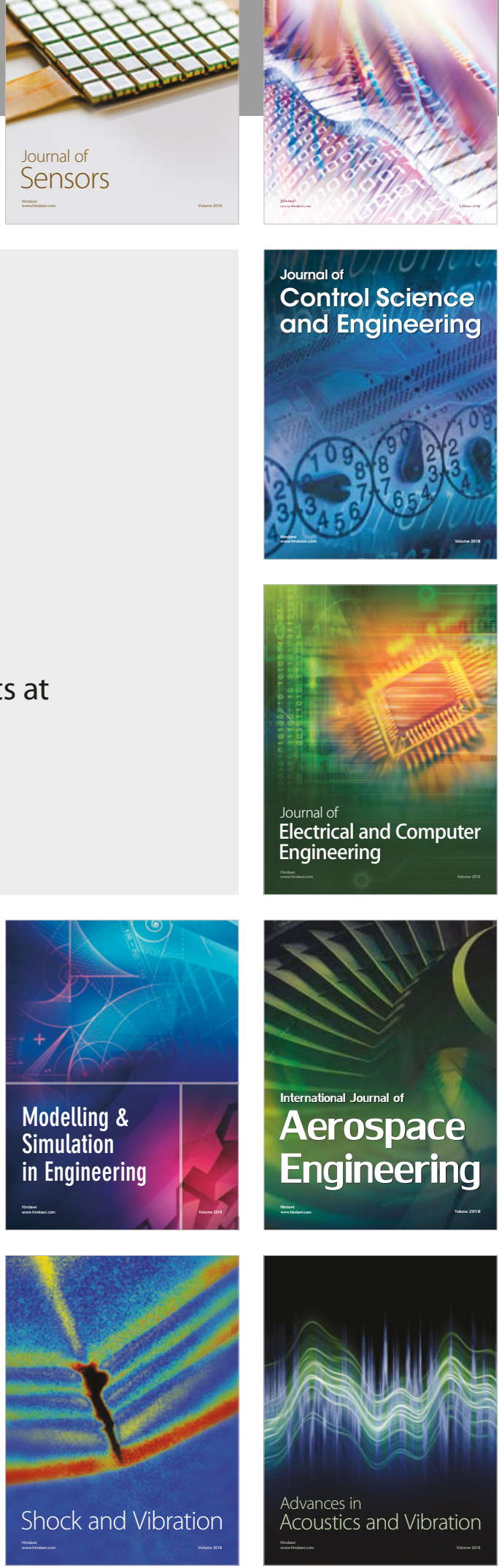